produced by the growth of a colony, which put a premium on mutants more fit than the mother colony for growth in the changed medium. This problem is that of formation of secondary colonies, which is familiar to bacteriologists.

Between the occurrence of a 'visible' mutation and the establishment of an actually visible patch of mutated mycelium there is a long way to go. In the initial stages, before the mutant has succeeded in securing a 'bridgehead', its survival or extinction is almost entirely a matter of chance. Once the bridgehead is secured, the systematic advantage or disadvantage of the mutant with respect to the parental strain determines the characteristics of the surfaces occupied by each, and therefore the ultimate fate of the mutant. Figs. 1, 4, 8 are examples of the simplest case, that of two strains one of which, with a higher growth-rate than the other, will ultimately supplant it. With certain obvious restrictions, these are models of the spread of a favourable gene in a population under the stress of natural selection alone. In Fig. 1 the mutant starts as a single individual in a large population; in Fig. 4 the mutant starts as 10 per cent of the initial population; in Fig. 8 two separate populations with different growthrates start at the same time and compete for the same territory : the boundary between them is a circle.

It is, however, as models of the accidental multiplication or elimination of genes, and its dependence on the size of the population, that mutants of moulds cain be most useful. Mixed colonies of strains with equal growth-rates are especially instructive, since in them the effect of natural selection between strains is nil, and that of chance alone stands out. A colony of this type, begun with great numbers of spores of each of two strains, shows (Fig. 5) in its early stages an apparently thorough mixture of both. Later on, the colony becomes made up of clearly distinct sectors each of either one or the other strain. As, with appropriate magnification, the early apparent mixture is seen to result from an alternation of very thin radial strips of each strain, the difference between earlier and later structure is simply a substitution of fewer sectors of great width for an initial very large number of sectors of very small width. Clearly a process of sorting out takes place: sectors which are too small in width either increase in width or disappear. The result is that any small apparently mixed arc in the young colony gives rise, after sufficient growth, to an arc in which one strain only is established to the exclusion of the other.

Here is an evident analogy with the Sewall Wright effect of the small size of populations upon the accidental fixation or extinction of genes. In the case of sexually reproducing species, the effect is due to the sampling nature of the reproductive process : accidental fluctuations in the frequency of a gene may, in a small population, reach the irreversible alternatives of either fixation or loss. In mould colonies a similar sampling operates. A hyphal tip in a colony of radius $r$ gives rise, on the average, to $r^{\prime} / r$ hyphal tips when the colony has grown to radius $r^{\prime}$. Accidents of growth, however, allow for fluctuations around this average: one hyphal tip will contribute more to the next 'generation', and another one less and leave no 'progeny'. In a small arc, with a small number of hyphal tips of two strains alternating, these fluctuations will be reversible and preserve the mixed constitution of the arc, so long as they do not happen to reach the irreversible state of all hyphæ being of one or the other strain. This will ultimately bring about the segregation of the two strains into 'sectors' of substantial size. To grasp fully the analogy between the Sewall Wright effect in sexually reproducing organisms and our example, one has to substitute a hyphal tip for a gene, and a small arc of the colony for a small population.

There are several possible ways of measuring the intensity of the sampling effects in moulds: unlike the sampling effect of sexual reproduction, its variance may be expected to depend on external conditions.

In conclusion, the remarkable diagrams that mould colonies may be made to produce offer a range of opportunities for the experimental approach to problems as apparently unrelated as morphogenesis and genetics of populations.

Wo wish to thank Miss A. H. Waddell for advice on the mathematical side, and Dr. W. M'Farkane, who carried out the irradiations at the Western Infirmary, Glasgow, for his most helpful contribution to our discussions. A research grant from the Carnegie Trust for the Universities of Scotland has covered part of the cost of materials.

\section{FOUNDATIONS OF ELECTRICAL MEASUREMENT*}

\author{
By DR. L. HARTSHORN \\ National Physical Laboratory
}

$T$ HE last two or three years have seen the publication of a surprising number of papers on the fundamental magnitudes of classical physics. Even more surprising is the divergence of opinion expressed on such well-worn themes as the precise significance of the magnitudes of everyday electricity and magnetism. Well-known expounders of these subjects at our universities have found one another unintelligible, and practising engineers and physicists have frequently been heard to ask if these concepts are really as doubtful as all that after half a century of experience and discussion.

There seems to be fairly general agreement that the working concepts should be defined by reference to the processes by means of which they are measured, but there is no sort of agreement as to what these processes are. It appears that there are many possible processes for each quantity and no agreement as to their relative status. One of the troubles is that the processes described are usually idealized. They have been described as 'theoretical experiments'. It is agreed that they have not actually been performed, but it is asserted that they could be performed in principle; for example, you take two point charges and measure certain distances and forces and so on, operations which appear to the experimentalist of to-day as practically impossible, and in the results of which he would have no confidence whatever.

An outstanding fact, and one which the experimentalist finds very reassuring, is that all the doubt and discussion surrounding these theoretical experiments seem to produce no repercussions on his own real experiments. His values are usually accepted without argument, understood and acted upon with useful results. $\mathrm{He}$ can only conclude that these theoretical operations about which there is so much argument cannot really be the basis of the laboratory work, which remains unaffected. The extent of our

* Based on a lecture to the Measurements Section of the Institution of Electrical Engineers delivered on May 19. 
agreement in practical matters shows that we must be working on some common basis. It is curious that it is so difficult to express it in terms on which we are all agreed.

Dr. Norman Campbell alone in recent years seems to have felt the necessity of maintaining a close and direct connexion between the principles and experimental laws which he enunciates as the basis of our measurements, and the methods that we actually employ in our everyday work. His work has, however, been strangely neglected in the recent discussions, and unfortunately, as he himself has pointed out, the matter is too complicated to be adequately covered by a few simple statements of the kind frequently offered. These are rightly used in introductory text-books, where it is expedient to conceal complexities until the more prominent ideas have been grasped, but when trying to find our basis the complexities must be faced. In these circumstances it would obviously be impossible to give here a precise statement of the position, but it may be of interest to indicate in broad outline some of its main features.

Measurement is essentially the derivation of numbers that are capable of representing physical properties in their relations to one another; and just as the various physical properties are defined by reference to the process adopted for their measurement, the numbers used in experimental physics are the symbols representing the results of the familiar physical or experimental operation of counting. The characteristic properties of numbers are the laws of addition, from which the other mathematical operations employed are derived, repeated addition leading to multiplication, the reverse operations providing subtraction and division, and so on. Thus the first condition to be satisfied in order that measurement may become possible is that some physical properties must obey laws that are analogous to the laws of addition for numbers. Another requirement is that operations must be devised that will enable us to judge of two objects, whether one is greater than, equal to, or less than, the other, in respect of some particular property. In short, judgments of equality and laws of physical addition form the basis of the whole problem.

It is generally agreed that in all our practical work we shall take length, mass and time as the primary properties, and probably everyone would agree with the statement that these quantities are all additive, though it is far from easy to say exactly what this statement means. Attempts to do so are apt to be tedious, but it is of interest to note that the measurement of these quantities depends only on the operations and principles mentioned above.

Length we define by reference to rigid rods. Judgments of equality are made by putting rods side by side and judging the alignment of fiducial marks or the coincidence of the planes of end-faces. Physical addition is performed with such rods by placing them end to end and in line. What justifies us in calling such a process addition ? Briefly, it has characteristics similar to those laid down in the laws of addition, commutative, associative and distributive, the essential ones being as follows. First, whenever we perform the operation an increase of length is brought about, as can be established by our ability to judge equality, etc. Secondly, if we apply the process to a number of such rods, the length of the combination is always the same, however the various operations are performed and in whatever order they are carried out.
It is easy to see that any property for which such a law of addition can be established becomes measurable if we can devise means for producing objects that are judged equal. We can by adding together many such objects produce a succession of systems of steadily increasing magnitude, which constitute a standard scale for that property. Any object to be measured is compared with the systems of the stand. ard scale, and when the two are judged to be equal, we count the number of original equal objects in the composite system. The familiar devices for reducing the labour of counting, and for increasing the precision of the process by including subdivisions in the standard scale, depend only on the same laws of addition.

The direct application of these laws to measurements of length of the highest precision is well illustrated by the use of sets of slip gauges. The physical addition of these gauges by their wringing surfaces is daily giving results consistent to one millionth of an inch. The application to mass is too obvious to need description, but time and frequency differ somewhat from the other primary magnitudes. In practical work we are solely concerned with periods of time, a concept based on recurrent phenomena. In rotating and vibrating systems, we recognize certain features which recur regularly. These recognizable features are regarded as defining instants separated by periods of time, and we can judge instinctively whether two such instants are simultaneous or whether one is 'before' or 'after' another. We choose some definite system as a standard and define its characteristic periods as equal intervals of time. Then the time between any two instants is, by definition, to be measured by counting the number of recurrences of the standard, after the first instant and before or simultaneous with the second. Clocks are standard periodic systems attached to mechanical counting devices or adding machines.

Frequency, which is perhaps more important than time in electrical work, is merely another aspect of the same phenomena: it is just the number of recurrences of the system in each standard period. A clock without its calculating machine is a frequency standard. It is well known to all wireless engineers that laws for the physical addition, subtraction, multiplication and division of frequency of certain periodic electrical systems have been experimentally established and that actual measurements of frequency are often made with the highest precision in virtue of these laws. It is a fortunate fact of Nature that these laws are consistent with our notion of time, which is additive by definition.

Angle also should be regarded as one of our primary properties. It is essentially a property denoting difference or change of direction, that is to say, a property characteristic of the faces of rigid bodies like crystals, or alternatively, one characteristic of the operation of rotation or of rotating systems. There is a common delusion that the measurement of angle is based on that of length, but both 'static' angles and angles of rotation are in practice measured fundamentally using only laws of addition, and there is close correspondence between the two. Anyone who has calibrated a divided circle will know that measurements of length play no essential part in the process, but that the essence of the matter is the continued addition of a number $(n)$ of equal rotations until exactly one complete turn has been made. Each of the equal rotations then bears the relation $1 / n$ to one complete revolution. Obviously angles 
of this kind are also measured whenever we count revolutions.

A most interesting illustration of the fact that angle of the other kind is measured fundamentally, and of its relation to rotation, is provided by a new system of angle gauges recently developed at the National Physical Laboratory by Dr. G. A. Tomlinson. They consist of pieces of hardened steel with plane faces $3 \frac{1}{2}$ in. $\times \frac{5}{8}$ in., inclined together at various angles and so truly plane that they can be wrung together to form combinations like length gauges. 'This is the process of addition. Equality of angles could be tested by the parallelism of corresponding faces, and thus starting with an arbitrary unit angle, standards of $1,2,2,5$, etc., or $1,2,4,8$, etc., could be established and any desired angle produced by addition. Tomlinson has, however, greatly simplified the practical problem by making use of the fact that if two equal standards are added with their directions reversed, their external faces are parallel, that is, their sum is zero : in other words, in this case physical subtraction as well as physical addition is possible, and therefore with a given number of gauges more combinations are possible. Tomlinson uses only twelve gauges of angles $3^{\prime \prime}, 9^{\prime \prime}, 27^{\prime \prime}, 1^{\prime}, 3^{\prime}, 9^{\prime}, 27^{\prime}$, and $1^{\circ}, 3^{\circ}, 9^{\circ}, 27^{\circ}, 41^{\circ}$, and with these he can quickly produce any angle between $0^{\circ}$ and $81^{\circ}$ to within $1 \frac{1^{\prime \prime}}{2}$ of its nominal value, some 97,000 possible values.

Measurements of angles of rotation can obviously be made with these gauges in conjunction with an auto-collimating microscope. The appropriate gauge is placed on the rotating member with one face perpendicular to the axis of collimation. The other face is, of course, not visible through the gauge, but it becomes available for inspection by wringing any plane reflecting surface on to it and arranging this surface so that it projects beyond the edge of the gauge and is thus exposed to the collimator. The rotating member is now turned until the second face becomes perpendicular to the collimator axis, as is shown by a zero reading in the eyepiece. The angle of rotation is then equal to that of the gauge. The gauges require no accurate centring, and provide a relatively simple method of measuring angle with the highest precision. Of the first four sets of gauges constructed at the National Physical Laboratory the average error was only $0 \cdot 2^{\prime \prime}$, and only three gauges out of forty-eight had errors so large as $0 \cdot 5^{\prime \prime}$.

Measurements of this kind, based solely on laws of physical addition and judgments of equality, are called by Norman Campbell fundamental or independent measurements. Not only are they employed for the most precise measurements of the primary mechanical properties; they are also employed for the most precise measurements of electrical properties. In the National Physical Laboratory, for example, they are employed for the basic measurements of resistance, capacitance and mutual inductance, and the laws of addition for these properties are the ones on which our electrical measurements primarily depend. Resistance is additive for conductors connected in series; capacitance for condensers connected in parallel; and mutual inductance for pairs of coils each consisting of a common primary coil and a secondary coil, when the secondary coils are connected in series. Standard scales for these quantities analogous to the scale of length are built up by the successive addition of resistors, condensers and inductors that are judged to be equal, the various bridges and balancing devices employed in making these judgments being detectors rather than measur- ing instruments, and serving only to amplify our senses.

Other electrical quantities which are additive are conductance, additive for conductors connected in parallel ; electromotive force, additive for cells in series; and current, additive in circuits in parallel connexion. These quantities can all be measured fundamentally with high precision over a limited range. For measurements over a wider range other processes of measurement are used. For conductance we usually base our measurements on the law connecting it with resistance, which it may be noted is very similar to that connecting frequency and time.

Fundamental measurement is obviously severely limited in its scope, since each property is measured independently of any knowledge of any other property. But it is the relations between different properties that give to physical science its great scope, and modern experimental technique consists largely of measurements based on the numerical laws expressing these relations. It is important, however, to recognize that these numerical laws are entirely dependent on the laws of fundamental measurement that have previously been established for certain of the properties. It is only possible here to consider the first two or three in the sequence of derived laws which forms the basis of our experimental work. The great bulk of the electrical apparatus used in Great Britain is calibrated by instrument makers by reference to standards calibrated at the National Physical Laboratory, and therefore measurements made with this apparatus must be based on the laws adopted at the National Physical Laboratory for standardizing purposes.

The first of these laws is that connecting mutual inductance $M$ and geometrical properties. It can be written

\[ M=\mu_{0} N, \]
where $N$ denotes Neumann's integral $\iint \frac{d s_{1} d s_{2} \cos \varepsilon}{r}$.

We have seen that for certain systems $M$ can be measured fundamentally. For many such systems $N$ can be expressed in terms of lengths and angles, which can also be measured fundamentally. It therefore becomes possible to establish with very high precision the law that for all mutual inductors constructed of non-magnetic material, $M$ and $N$ are proportional to one another, the proportionality constant $\mu_{0}$ depending only on the units adopted for $M$ and $N$ respectively. It can also be established that for inductors made of magnetic material the law becomes $M=\mu N$, where $\mu$ is constant for any one material, but varies with change of material. This law therefore provides a process for the measurement of the property $\mu$ characteristic of magnetic materials. It is the law defining magnetic permeability, and most of the usual methods of measuring permeability are directly based on it. The constant $\mu_{0}$ must now be interpreted as the permeability of all non-magnetic materials. We can regard it as having the dimensions 1 in inductance, and -1 in length, if we find the practice useful, and it is convenient to write its value as $10^{-8}$ henry per cm. The unit of inductance, the henry, has of course been fixed by assigning this value to $\mu_{0}$.

Our next law is that of the electrodynamometer, which forms the working basis of most of our measurements of current. It can bo written

$$
F=a I^{2} \frac{d M}{d z}
$$


where $F$ denotes the mechanical force between two coils when each carries the same current $I, M$ denotes the mutual inductance between the coils, and the $z$-axis is the direction of the force. This law has been established with the highest precision for the special case of the current balance built of non-magnetic material, the force $F$ being determined by weighing, in terms of mass, and the quantity $\frac{d M}{d z}$ by application of the law of mutual inductance in terms of the geometrical property $\frac{d N}{d z}$. I can be measured fundamentally over a limited range, and thus the dynamometer law is established by means of the current balance in the form

$$
m g=a I^{2} \mu_{0} \frac{d N}{d z} .
$$

The proportionality constant $a$, unlike $\mu_{0}$ in the previous law, appears to be characteristic of no important property. We therefore choose our unit of $I$ so that $a$ takes the value of unity and disappears from our working equation, which becomes

$$
F=I^{2} \frac{d M}{d z}
$$

Next in the sequence is Ohm's Law, the law of the potentiometer, which can be established in the form

$$
E=b I . R,
$$

since both $E$ and $R$ can be measured fundamentally, while $I$ can be measured both fundamentally and by means of the dynamometer law. The constant $b$ appears to be characteristic of no important property and is therefore suppressed by a suitable choice of units for $E$ and $R$.

It is impossible to pursue the subject further here, but the examples given are suficient to show the kinds of law that form our real foundations at the present time. These foundations change to some extent with changing technique. The basic laws are those of the instruments and operations which have enabled us to correlate our experiences with the highest precision, and have therefore been used in establishing our working standards. At the present time, alternating currents are employed for precision electrical work to an increasing extent, and therefore some of the laws concerning alternating currents are of basic importance. Note that the electrodynamometer law provides us with a means of measuring alternating current as well as direct current, and then by means of the electrostatic voltmeter we can extend Ohm's Law to certain classes of conductors, namely, resistors. Thus we can define and measure alternating potential difference. Faraday's Law of Induction can then be established in the form, in which it states the relation between the alternating potential difference at the secondary terminals of a mutual inductor $M$ of which the primary carries a current $I$. Adopting the usual vector notation, we establish the law

$$
E=k M \omega I,
$$

and then choose the unit of $E$ so that $k=1$. The constant $b$ which appeared in Ohm's Law is then suppressed by a suitable choice of the unit of resistance.

It is unfortunate that this system of laws, upon which our experimental work is based, does not readily link up to form a simple logical development of the theory of the subject; but once we have recognized the great difference between the basic experimental laws and the theoretical relations which have been devised as the simplest easily workable system consistent with these laws, we are prepared to find differences of opinion as to which theoretical scheme can be used with the least risk of confusion.

\section{PSYCHOLOGICAL ASPECTS OF MORAL PROGRESS}

A WIDESPREAD readiness to re-examine human values makes it particularly appropriate at the present time to ask whether recent scientific work can add to our understanding of moral advance. This was the question taken up in a discussion on "Psychological Aspects of Moral and Social Progress" at a meeting of the British Psychological Society on September 23, in which the chief speakers were Prof. J. C. Flugel, Prof. Karl Mannheim and Dr. R. H. Thouless.

In opening the discussion, Prof. Flugel suggested that, as a first approximation, we may identify eight mental tendencies exhibited in what we regard as moral progress. Once recognized, they can be illustrated in three fields of comparison: they differentiate the mind of the infant and child from the more mature mind, the abnormal (pathological) mind from the healthy mind, and the primitive from the civilized culture. These eight tendencies may be briefly stated.

(1) There is a trend away from ego-centricity and towards sociality, an increasing readiness to subordinate one's own immediate requirements to the needs of one's fellows, and to find satisfaction in the well-being of groups ever more broadly conceived, so that what starts as a concern for a few family associates may develop into loyalty to a nation, a church, or the whole human race. Among the psychological mechanisms involved in this process, an important part is played by vicarious satisfaction; our interest goes out to people very different from ourselves because they represent some of what William James called the 'potential selves' which we all give up in becoming our own one self.

(2) Progress towards greater consciousness, away from the control of conduct and feeling by unconscious urges, also appears in the advance towards individual maturity and civilized social organization. Progress along this line is pre-eminently the aim of psycho-analysis. At the same time, it must be recognized that, conscious scrutiny having thoroughly performed its task, much behaviour has to be relegated to the habitual and the automatic. Individual conscious life is supported by a mass of habits, reflexes, and other non-conscious determining tendencies. In social life, traditions and conventions have a similar role. Their value lies in the economy they effect, their danger in that they may grow antiquated and ill-adapted; but they must always be necessary in mental and social organization, simply because the span of consciousness at any one time is strictly limited.

(3) The trend towards realistic thinking, away from dreaming and 'wishful thinking' in individual life, and away from magic in social life, is a third mark of adwance. Science, Freud has suggested, is essen- 